EPJ Web of Conferences 116, 03003 (2016)

DOI: $10.1051 /$ epjconf/201611603003

(C) Owned by the authors, published by EDP Sciences, 2016

\title{
The ARIANNA Hexagonal Radio Array - performance and prospects
}

\author{
Allan Hallgren ${ }^{a}$ for the ARIANNA collaboration \\ Department of Physics and Astronomy, Uppsala University, Box 516, 75120 Uppsala, Sweden
}

\begin{abstract}
The origin of the highest energy cosmic rays at $\sim 10^{20} \mathrm{eV}$ is still unknown. Ultra-high energy neutrinos from the GZK process should provide information on the sources and their properties. A promising and cost effective method for observing GZKneutrinos is based on detection of Askaryan radio pulses with antennas installed in ice. The ARIANNA project aims at instrumenting a $36 * 36 \mathrm{~km}^{2}$ large area on the Ross Ice Shelf with an array of radio detection stations. The deployment of a test system for ARIANNA, the Hexagonal Radio Array (HRA), was completed in December 2014. The three first stations were installed in 2012. Solar panels are used to drive the $<10 \mathrm{~W}$ stations. The system hibernated at sunset in April and all stations returned to operation in September. The site is essentially free of anthropogenic noise. Simple cuts eliminate background and provides for efficient selection of neutrino events. Prospects for the sensitivity of the full ARIANNA array to the flux of GZK neutrinos are shown.
\end{abstract}

\section{Introduction}

The origin of the very highest energy cosmic rays remains enigmatic still more than 100 years after the discovery of cosmic rays by V. Hess in 2012. A cut-off in the spectrum is observed and may correspond to interactions with the cosmic microwave background as suggested by Greisen, Zatsepin and Kuzmin (GZK) [1, 2]. Production of neutrinos with energies around $10^{18} \mathrm{eV}$ would then occur. Detection of GZK-neutrinos requires surveillance of a large amount of target material. Observation of radio pulses from the Askaryan effect $[3,4]$ using antennas embedded in Antarctic ice or firn promises a cost effective technique to achieve the goal of GZK-neutrino detection. ARIANNA (ㅍntarctic $\underline{\text { Ross }}$ IceShelf $\underline{\text { ANtenna }} \underline{\text { Neutrino }} \underline{\text { Array) }}$ is a proposed detector system for this purpose. Determination of the flux of GZK-neutrinos, their spectral properties, directional and temporal distributions would provide crucial information on the high energy universe. Further, physics beyond the standard model may be revealed if the flux permits to determine e.g. the cross section at these extreme energies. Even if no GZK-neutrinos are found limiting the flux below most current models will provide important information on the origin of the highest energy particles observed.

The detection mechanism relies on coherent emission of a sub-nanosecond Cherenkov pulse at radio wavelengths. The particle shower caused by the neutrino-interaction develops a negative charge excess as it travels through the medium. Constructive interference of Cherenkov radiation generates a

\footnotetext{
a e-mail: allan.hallgren@physics.uu.se
}

This is an Open Access article distributed under the terms of the Creative Commons Attribution License 4.0, which permits unrestricted use, distribution, and reproduction in any medium, provided the original work is properly cited. 


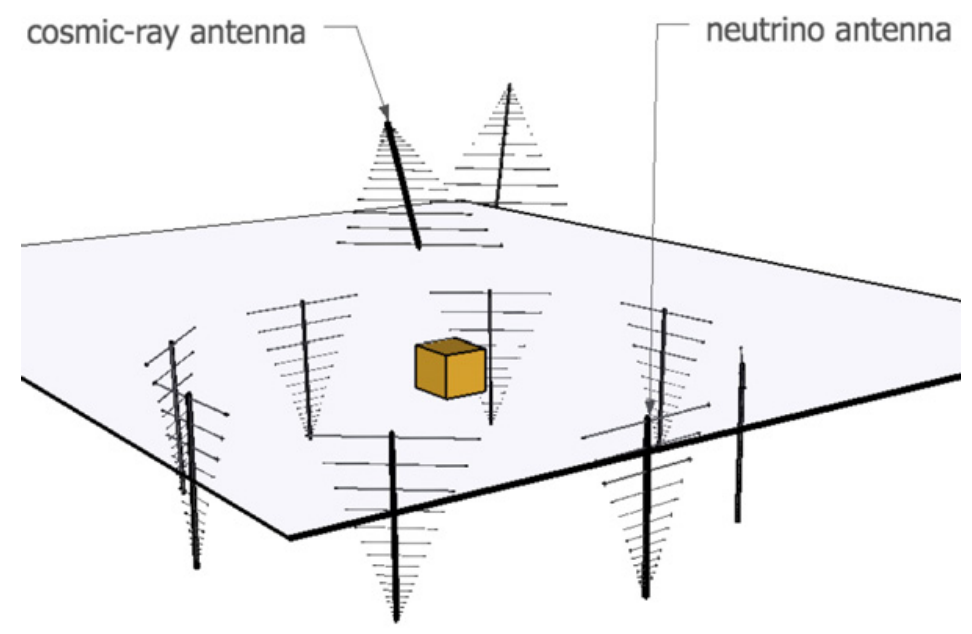

Figure 1. Conceptual design of an ARIANNA station with eight downward pointing antennas just below snow surface and two upward tilted antennas for identification of radio signals from cosmic rays. All antennas are connected to the station central readout system. Power and communication systems are not shown. The prototype stations in the Hexagonal Radio Array is a reduced version with 4 downward directed antennas.

sub-nanosecond pulse in radio-wavelengths as predicted by G. Askaryan. This radio pulse generation and its properties have been experimentally verified with good agreement using electron bunches stopped in e.g. blocks of ice [5]. The signal generation and pulse properties behave as expected, allowing for simulations of all the variations of pulses that a detector will have to efficiently identify.

\section{The ARIANNA development work}

The full ARIANNA system would comprise 1236 independent stations distributed over an area of $36 \mathrm{~km} * 36 \mathrm{~km}$ on the immense Ross Ice-Shelf [6]. Each station has in the conceptual design eight downward directed antennas deployed just below the snow surface and two upward antennas for suppression of background from radio pulses induced by cosmic rays, Fig 1 . The flat ice to sea-water interface reflects the downward directed part of the Askaryan radio pulse. Most of the neutrino events recorded by ARIANNA will be seen through the reflected signal. The proposed location is $\sim 120 \mathrm{~km}$ from the McMurdo station, a US driven base for research in Antarctica. This remote site is essentially free of anthropogenic radio noise due to the distance and to screening by mountain ridges.

\subsection{The Hexagonal Radio Array}

The Hexagonal Radio Array (HRA) serves as a prototype system for ARIANNA. The seven HRA stations contain a reduced number of four antennas per station. Three stations were installed in 2012, the remaining four were deployed in December 2014. The equipment for the last four stations was prepared and planned for deployment in 2013 but due to US Government shutdown the installation was postponed. Only some maintenance work was possible in 2013. Data taken by HRA-3 thereafter, Jan-April 2014, have been analysed for content of neutrino events. The closing date for this analysis was set by hibernation of the solar powered stations. No v-event was found, but more importantly demonstrated that three simple cuts were sufficient to remove all background events [6]. Autocorrelation, where a pulse is correlated with a time shifted copy of itself, is a simple test that removes much of the thermal noise, showing strong correlation only at a time shift of zero. Pulses from $v$-events, as simulated 
through the electronics chain, show negative correlation when shifted a few nanoseconds and can thus be separated. The second cut analyse the frequency power distribution, neutrino events have a broader distribution than noise events that typically have power in only few of the frequency bins. The third and final selection criterion compares the recorded event to a library of events simulated with different observational conditions and the best correlation is used for selecting neutrino candidate events. The efficiency of the analysis was above $80 \%$ for a typical GZK spectrum and the flux limit obtained from three stations in three months of data was $\mathrm{E}^{2} \phi(\mathrm{E}) \sim 2 \cdot 10^{5} \mathrm{GeVcm}^{2} \mathrm{~s}^{1} \mathrm{sr}^{1}$ [6]. This result is promising. To asses rarer types of background more stations and live-time is needed and is forthcoming from the now completed HRA.

The full HRA system also serves to test and verify the functionality of all technical systems [7]. An important challenge is the power system. Solar power panels of are used together with lithium batteries for buffering. The first generation of batteries used have proven unreliable, and some of the stations were run with non-functional batteries or without batteries. If no battery is available the fraction of the day the station is alive gradually decreases from mid-January to mid-April. If a working battery is present full functionality can be maintained until mid-April when the Sun is only a few degrees above horizon and the power generated becomes too low. All stations came back to operation during September when the Sun came back over horizon. The resulting live-time fraction achieved is thus $\sim 60 \%$ for stations with working buffering capacity [8]. This live-time fraction is included in the sensitivity estimates for ARIANNA below.

A more reliable Li-battery with lower but sufficient capacity $(12 \mathrm{~V}, 20 \mathrm{Ah})$ has been found and extensively tested in the lab. Additionally a better charge control unit has been evaluated, in particular the noise generated when switching is substantially reduced. The batteries and charge controller will therefore be exchanged on all installed stations in the coming 15/16 field season. The smaller dimensions allow these components to be included in an RF-tight box. This is likely to remove a large part of noise seen when solar power generation is marginal.

The trigger rate has been verified to be low in all stations. After proper adjustment the rate is $\sim$ few ${ }^{*} 10^{-2} \mathrm{~Hz}$. The electronics gain is known to rise with decreasing temperature, thus trigger level adjustments are required to maintain the rates. The electronics box is buried in the firn and the temperature changes only slowly. Periods of strong winds are seen as increased trigger rate, but these events are all eliminated in the template correlation analysis [9].

Tests are done with RF pulses emitted downwards from known positions at surface and detected in the array after bounce on the ice-water interface. The RF pulse has thus propagated $\sim 1.1 \mathrm{~km}$ through the ice. The reflected pulses maintain their polarization. The time differences between the signals' received in the different antennas permit determination of the direction of the incoming radiation. A likelihood fit to the direction gives a resolution of $\sim 1^{\circ}$ [9]. The direction of the incoming neutrino can be determined from the direction to the event paired with the measured polarisation. The likelihood can be used in the event selection as shown in Fig. 2. This was not used in the analysis of HRA-3 data [6] but will allow further noise suppression.

One station was installed with upward tilted antennas to verify the signal amplitudes recorded for cosmic ray events. This station detected signals from a coronal mass ejection from the Sun on Dec 20, and the subsequent auroras activity a few days later. Noise from the Galactic centre is also visible in forced readouts with a daily modulation and with the expected power ratio between upward and downward antennas [8]. These observations demonstrate that the system sensitivity to RF signals is according to expectations. The characteristics of the radiation, e.g. the frequency content, show that this type of background can be readily discarded from neutrino candidate events. 

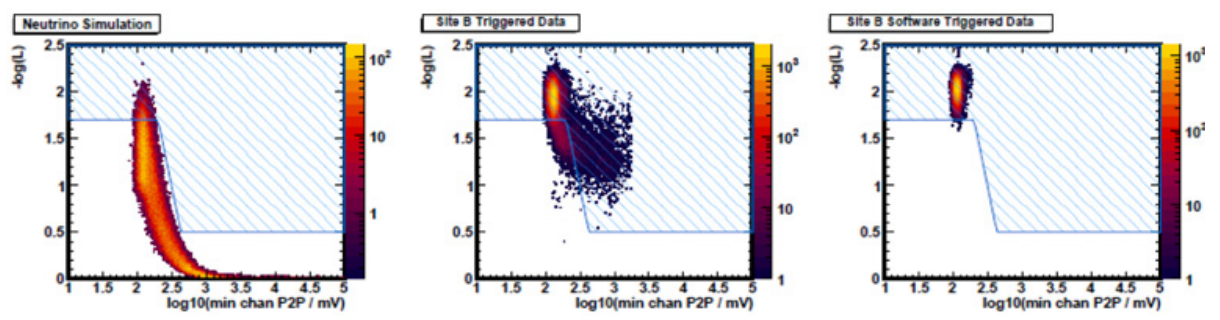

Figure 2. The directional fit likelihood plotted versus the peak to peak amplitude for simulated neutrino events (left), triggered data in HRA (middle) and pure thermal noise events (right). All plots are on events surviving the cut on distribution of power [9]. The passing fraction is $96 \%$ for neutrino events if the indicated cut is used.

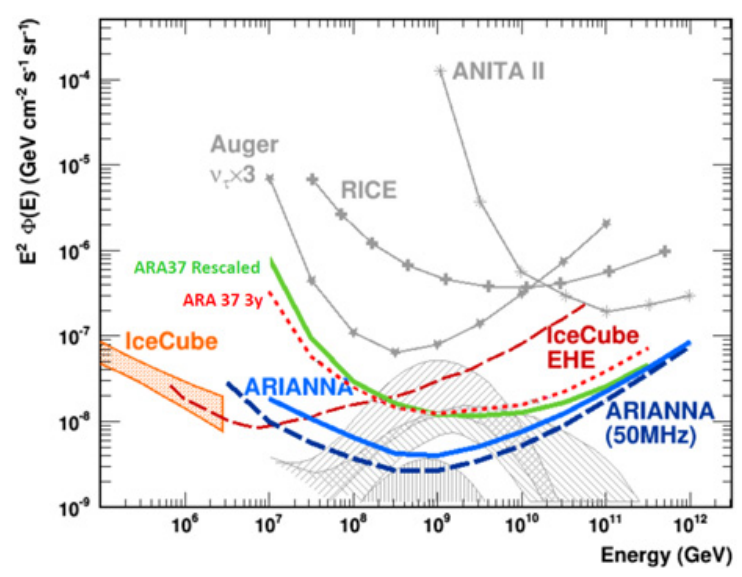

Figure 3. Projected 5 calendar year sensitivities for ARIANNA (36*36 stations) and $50 \mathrm{MHz}$ ARIANNA option. For ARA37 the projected 3 year sensitivity from [10] is given as well as a rescaled 5 year estimate, see text for explanation. The 5 year sensitivity of the IceCube EHE analysis is shown as well as a selection of current limits and range of predictions for the flux of GZK-neutrinos.

\section{Projected performance of ARIANNA}

The $90 \%$ CL sensitivity of the full $36 * 36$ station ARIANNA array has been estimated in the standard configuration with $100 \mathrm{MHz}$ antennas and with an alternative $50 \mathrm{MHz}$ antenna, as shown in Fig. 3. The performance of the $50 \mathrm{MHz}$ option, including noise, will be tested in the near future as it offers an interesting possibility to enhance the sensitivity at lower energies. This sensitivity corresponds to $\mathrm{N}_{\text {bck }}=0$ and $\mathrm{N}_{90}=2.3$ events. To compare to the sensitivity of the proposed ARA37 array two curves are shown. The curve labelled "ARA37 3yr" is taken from [10]. The curve labelled "ARA37 Rescaled" is for a 5 calendar year period but includes reduction in analysis efficiency and live time derived from [10]. This curve is further recalculated to be comparable to the ARIANNA curves by using e.g. the same cross sections and statistical methods for determination of the differential sensitivity [11].

The Askaryan RF pulse is polarized with the shower direction included in the E-field plane. The ARIANNA antennas measure polarization and this combined with the direction to the interaction point (described above) allows the $v$-direction to be resolved. Simulations show that the expected resolution is $\sim 2.5-3$ degrees [6]. The sky coverage starts at $-90^{\circ}$ declination and has an energy dependent cutoff somewhat below horizon. Due to the ARIANNA position at about $78^{\circ} \mathrm{S}$ a larger part of the sky is visible than would be possible for a detector located at $90^{\circ} \mathrm{S}$. This location results in that a point source 
of GZK-neutrinos will be seen with a modulated viewing angle, allowing for additional information on cross section and spectral properties.

The ARIANNA HRA has now demonstrated a cost effective possibility to build an instrument capable of measuring the flux of GZK-neutrinos, often dubbed "guaranteed". This would provide conclusive information on the yet enigmatic origin of the cosmic rays with the highest energies observed. It is time to start building!

\section{References}

[1] K. Greisen, Phys. Rev. Lett. 16, 748 (1966)

[2] G.T. Zatsepin and V.A. Kuzmin, JETP Lett. 4, 78 (1966)

[3] G.A. Askaryan, JETP 14, 441 (1962)

[4] G.A. Askaryan, JETP 21658 (1965)

[5] P. Gorham et al. (ANITA Collaboration), Phys. Rev. Lett. 99, 171101 (2007)

[6] S.W. Barwick et al. [arxiv: 1410.7352], Astroparticle Phys. 7012 (2015)

[7] S.W. Barwick et al. [arxiv: 1410.7369], IEEE TNS 62, 2202 (2015)

[8] A. Nelles and C. Persichelli for the ARIANNA Collaboration, PoS(ICRC2015) 1087 (2015)

[9] C. Reed for the ARIANNA Collaboration, PoS(ICRC2015) 1149 (2015)

[10] P. Allison et al. (ARA collaboration), [arxiv: 1507.08991v2]

[11] C. Reed, S.W. Barwick, Private communication 